\title{
Phase transitions of geometrically frustrated mixed spin-1/2 and spin-1 lsing-Heisenberg model on diamond-like decorated planar lattices
}

\author{
Lucia Gálisovấ1 ${ }^{1}$, Jozef Strečka $a^{2}$ \\ ${ }^{1}$ Department of Applied Mathematics, Faculty of Mechanical Engineering, Technical University, Letná 9 , \\ 04200 Košice, Slovak Republic \\ 2 Department of Theoretical Physics and Astrophysics, Faculty of Science, P. J. Šafárik University, Park \\ Angelinum 9, 04001 Košice, Slovak Republic
}

Received July 26, 2010, in final form October 21, 2010

\begin{abstract}
Phase transitions of the mixed spin-1/2 and spin- 1 Ising-Heisenberg model on several decorated planar lattices consisting of interconnected diamonds are investigated within the framework of the generalized decoration-iteration transformation. The main attention is paid to the systematic study of the finite-temperature phase diagrams in dependence on the lattice topology. The critical behaviour of the hybrid quantum-classical Ising-Heisenberg model is compared with the relevant behaviour of its semi-classical Ising analogue. It is shown that both models on diamond-like decorated planar lattices exhibit a striking critical behaviour including reentrant phase transitions. The higher the lattice coordination number is, the more pronounced reentrance may be detected.
\end{abstract}

Key words: Ising-Heisenberg model, decoration-iteration transformation, geometric frustration, reentrant phase transitions

PACS: $05.50 .+q, 75.10 . H k, 75.10 . J m, 68.35 . R h$

\section{Introduction}

Low-dimensional quantum spin models provide an excellent playground for theoretical studies of cooperative and quantum phenomena. It should be pointed out that quantum spin systems, which are prone to a mutual interplay of geometric frustration, thermal and quantum fluctuations, reveal the most remarkable properties [1-3]. Under this specific constraint, one often encounters a strikingly rich physics manifested in diverse unusual ordered and disordered ground states [1], the order-from-disorder effect [1 3], the chirality [4, 5], the enhanced magnetocaloric effect [6 9] or the quantized magnetization plateaus observable at low enough temperatures [2, 10].

It is also well-known dictum that the theoretical investigation of quantum spin models is frequently accompanied by rather cumbersome and sophisticated mathematics, which precludes an exact treatment of the most (even simple-minded) spin systems. Hence, it follows that one usually has to rely on an application of some approximative method(s) when treating the vast majority of low-dimensional quantum spin models. However, the approximative methods might have a profound deficiency in that they need not be reliable enough in order to decide whether the observed (often subtle) quantum phenomena are of a real physical significance or they merely arose as an artefact of the applied approximation(s). From this point of view, it is highly desirable to search for artificial but exactly tractable quantum spin models, which might display non-trivial quantum effects without a danger of over-interpretation inherent in any approximation.

The hybrid Ising-Heisenberg models on diamond-like decorated lattices [11 19] belong to the simplest exactly solved quantum spin models, which were envisaged for describing lattice-statistical systems composed of the semi-classical Ising and the quantum Heisenberg spins. These simplified quantum-classical models can be rigorously treated within the framework of generalized decorationiteration mapping transformation [20 22], because the nodal Ising spins represent a barrier for 
quantum fluctuations that are consequently restricted to elementary diamond-shaped units only. It is also worth mentioning that the Ising-Heisenberg models on the diamond-like decorated lattices have turned out to be a very useful testing ground for elucidating several typical quantum features. Indeed, these interesting but still exactly tractable spin systems may exhibit diverse quantum ordered and disordered ground states [11 15, 18, 19], the multi-step magnetization process with quantized intermediate magnetization plateaus [14, 17, 18], the enhanced magnetocaloric effect [14, [18], as well as the non-trivial criticality [13, 16, 19].

In our recent works [11, 16, 19], we have examined in detail the ferromagnetic mixed spin$1 / 2$ and spin-1 Ising-Heisenberg model on several diamond-like decorated planar lattices. It has been demonstrated that this hybrid classical-quantum model exhibits a rather rich ground-state and finite-temperature phase diagrams on account of the competition between the easy-axis Ising and the easy-plane Heisenberg interaction. The main purpose of the present work is to investigate critical properties of the antiferromagnetic mixed spin-1/2 and spin-1 Ising-Heisenberg model on diamond-like decorated planar lattices, which might even display more complex and non-trivial criticality owing to an interplay of the geometric frustration, thermal and quantum fluctuations as evidenced by our preliminary ground-state analysis [15].

The organization of this paper is as follows. In the next section, we shall define the mixed spin-1/2 and spin-1 Ising-Heisenberg model on diamond-like decorated lattices and we shall also briefly review the most important steps of an exact calculation based on the decoration-iteration procedure. The section 3 deals with a discussion of the most interesting numerical results for finitetemperature phase diagrams and temperature variations of the total magnetization both for the classical-quantum Ising-Heisenberg model and its semi-classical Ising analogue. In this section, our main attention will be focused on a possibility of observing reentrant phenomenon. Finally, some concluding remarks are drawn in section 4

\section{Formulation}

Let us consider two-dimensional lattices composed of inter-connected diamonds as is illustrated in figure 1 for honeycomb, square and triangular lattices. In this figure, the empty circles denote lattice positions of the Ising spins $\sigma=1 / 2$ that interact with other spins through the interaction $J_{\mathrm{I}}$ and the full ones represent lattice positions of the decorating Heisenberg spins $S=1$ that interact among themselves via the anisotropic XXZ coupling $J_{\mathrm{H}}(\Delta)$. Note that the parameter $\Delta$ allows to control the interaction $J_{\mathrm{H}}$ between the easy-axis $(\Delta<1)$ and easy-plane $(\Delta>1)$ type, as well as, to obtain the Ising model as a special limiting case when assuming $\Delta=0$. Providing that the parameter $D$ stands for the axial zero-field splitting (AZFS) parameter [23, 24], which acts on the decorating Heisenberg spins $S=1$ only, the total Hamiltonian of the investigated model can be

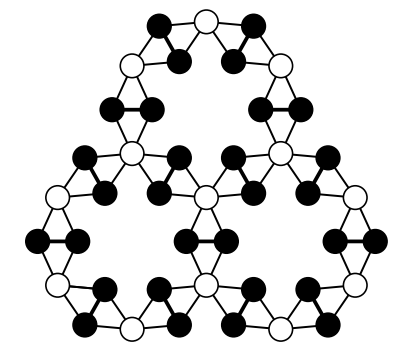

(a)

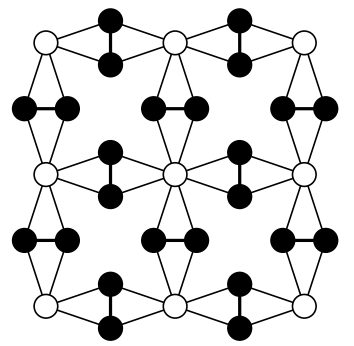

(b)

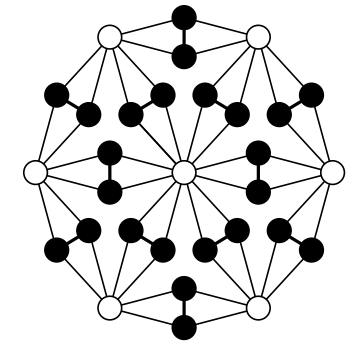

(c)

Figure 1. The mixed spin-1/2 and spin-1 Ising-Heisenberg model on diamond-like decorated honeycomb (figure 1a), square (figure 1b) and triangular (figure 15) lattices. White circles represent the nodal Ising spins $\sigma=1 / 2$ and black ones denote the decorating Heisenberg spins $S=1$. 
written as

$$
\hat{\mathcal{H}}=-J_{\mathrm{H}} \sum_{(i, j)}^{N q / 2}\left[\Delta\left(\hat{S}_{i}^{x} \hat{S}_{j}^{x}+\hat{S}_{i}^{y} \hat{S}_{j}^{y}\right)+\hat{S}_{i}^{z} \hat{S}_{j}^{z}\right]-J_{\mathrm{I}} \sum_{(i, n)}^{2 N q} \hat{S}_{i}^{z} \hat{\sigma}_{n}^{z}-D \sum_{i=1}^{N q}\left(\hat{S}_{i}^{z}\right)^{2}
$$

Here, the first summation is carried out over all interactions between pairs of the nearest-neighbouring Heisenberg spins, the second summation takes into account the interaction between the nearest-neighbouring Ising and Heisenberg spins and the last summation runs over all lattice sites occupied by the decorating Heisenberg spins. The spin variables $\hat{\sigma}_{n}^{z}$ and $\hat{S}_{i}^{\gamma}(\gamma=x, y, z)$ denote spatial components of the spin- $1 / 2$ and spin- 1 operators located at $n$th and $i$ th lattice position, respectively. Finally, $N$ represents the total number of the Ising spins, and $q$ is the coordination number of the lattice, which implies that the total number of all spins (lattice sites) is $N_{\text {tot }}=$ $N(q+1)$. It is worth mentioning that the quantum-classical model defined in this way is exactly solvable within the framework of a generalized decoration-iteration mapping transformation 20 22] (for more computational details see our recent work [19], where we presented a detailed procedure for an exact solution of this model). As a result of this mapping, one obtains a simple relation between the partition function $\mathcal{Z}$ of the investigated mixed spin- $1 / 2$ and spin- 1 Ising-Heisenberg model on diamond-like decorated lattices and the partition function $\mathcal{Z}_{\text {Ising }}$ of the simple spin- $1 / 2$ Ising model on corresponding undecorated lattices with the nearest-neighbour coupling $R$ :

$$
\mathcal{Z}\left(T, J_{\mathrm{I}}, J_{\mathrm{H}}, \Delta, D\right)=A^{N q / 2} \mathcal{Z}_{\mathrm{Ising}}(T, R)
$$

Note that both mapping parameters $A$ and $R$ are unambiguously determined by a self-consistency condition of the applied decoration-iteration mapping transformation and their explicit forms are given by equations (5)-(7) of reference [19].

At this stage, it is worthwhile to remark that the mapping relation (2.2) is universal and valid regardless of the lattice topology and spatial dimensionality of the model system. In addition, it also permits a rather comprehensive analysis of its critical behaviour as well as some basic thermodynamic quantities. Indeed, it directly follows from equation (2.2) that the investigated mixed-spin Ising-Heisenberg model becomes critical if and only if the spin- $1 / 2$ Ising model with the effective coupling $R$ on the corresponding undecorated lattice becomes critical as well. Bearing this in mind, the exact critical temperature of the spin-1/2 and spin-1 Ising-Heisenberg model on diamond-like decorated honeycomb, square and triangular lattices can be straightforwardly obtained from the conditions

$$
\beta_{\mathrm{c}} R=2 \ln (2+\sqrt{3}), \quad \beta_{\mathrm{c}} R=2 \ln (1+\sqrt{2}), \quad \beta_{\mathrm{c}} R=\ln 3,
$$

that represent exact relationships between the effective temperature-dependent coupling $\beta R$ and relevant critical temperatures of the simple spin-1/2 Ising model on a corresponding undecorated honeycomb [25], square [26] and triangular [27] lattices, respectively. In the above, $\beta_{\mathrm{c}}=1 /\left(k_{\mathrm{B}} T_{\mathrm{c}}\right)$ and $T_{\mathrm{c}}$ is the critical temperature of the studied quantum mixed-spin Ising-Heisenberg model. Similarly, the sub-lattice and total magnetization can also be derived from the exact mapping equivalence (2.2) between the partition functions $\mathcal{Z}$ and $\mathcal{Z}_{\text {Ising. More specifically, by combining }}$ equation (2.2) with the exact mapping theorems developed by Barry et al. 28 30] and the generalized Callen-Suzuki spin identity [31 33], the sub-lattice magnetization $m_{\mathrm{i}}^{z}, m_{\mathrm{h}}^{z}$ reduced per one Ising and Heisenberg spin, respectively, can be directly computed from the precise relations:

$$
\begin{aligned}
& m_{\mathrm{i}}^{z} \equiv\left\langle\hat{\sigma}_{k 1}^{z}\right\rangle=\left\langle\hat{\sigma}_{k 1}^{z}\right\rangle_{0} \equiv m_{0}, \\
& m_{\mathrm{h}}^{z} \equiv\left\langle\hat{S}_{k 1}^{z}\right\rangle=4 m_{0} F\left(\beta J_{\mathrm{I}}\right),
\end{aligned}
$$

where $F(x)=\left[\exp \left(2 \beta D+\beta J_{\mathrm{H}}\right) \sinh (2 x)+\exp (\beta D) \sinh (x) \cosh \left(\beta J_{\mathrm{H}} \Delta\right)\right] / W_{1}$, the parameter $W_{1}$ is defined by equation (6) of reference [19] and the symbols $\langle\ldots\rangle$ and $\langle\ldots\rangle_{0}$ represent standard canonical averages performed over the ensemble defined by the mixed-spin Ising-Heisenberg model on the diamond-like decorated lattice and the spin-1/2 Ising model on the corresponding lattice, respectively, and $m_{0}$ labels the single-site magnetization of the corresponding Ising model. In view of 
this notation, the total magnetization reduced per spin of the investigated lattice, which represents the order parameter of the mixed-spin Ising-Heisenberg model on diamond-like decorated lattices, can be expressed as follows: $m=\left(m_{\mathrm{i}}^{z}+q m_{\mathrm{h}}^{z}\right) /(q+1)$.

\section{Results and discussion}

In this part, let us proceed to a discussion of the most interesting results obtained for the mixed spin-1/2 and spin-1 Ising-Heisenberg model on diamond-like decorated honeycomb, square and triangular lattices. Before doing this, however, it is worth mentioning that all the results obtained in section 2 are universal since they hold true regardless of whether ferromagnetic or antiferromagnetic interactions $J_{\mathrm{I}}$ and $J_{\mathrm{H}}$ are assumed, and are independent of the lattice topology or spatial dimensionality of the investigated system.

Moreover, the effective nearest-neighbour interaction $R$ of the spin-1/2 Ising model on the corresponding undecorated lattice is invariant under the transformation $J_{\mathrm{I}} \rightarrow-J_{\mathrm{I}}$ [see equations $(5)-(7)$ in reference [19]]. This observation leads to the conclusion that the critical temperature as well as other thermodynamic quantities of the model under investigation remain unchanged under the change of the nature of the Ising interaction $J_{\mathrm{I}}$. Indeed, a change of the ferromagnetic interaction $J_{\mathrm{I}}>0$ to the antiferromagnetic one $J_{\mathrm{I}}<0$ causes just a trivial change in the local alignment of the nodal Ising spins with respect to their nearest Heisenberg neighbours. By contrast, there are some fundamental differences between magnetic behaviour of models with distinct nature of the Heisenberg interaction $J_{\mathrm{H}}(\Delta)$ (see our preliminary reports [11, 15, 16]). Taking this fact into account, we have restricted our recent work [19] to the particular case with the ferromagnetic Heisenberg and Ising interactions $\left(J_{\mathrm{H}}>0, J_{\mathrm{I}}>0\right)$ only. Besides a rather complex ground state composed of two unusual quantum phases, a striking critical behaviour including reentrant phase transitions with two or three consecutive critical points has been discussed in this work. With this background, our attention in this paper will be focused on the analysis of finite-temperature behaviour of the mixed spin-1/2 and spin-1 Ising-Heisenberg model on diamond-like decorated planar lattices, in which both the interaction constants $J_{\mathrm{I}}$ and $J_{\mathrm{H}}$ are supposed to be antiferromagnetic $\left(J_{\mathrm{I}}<0, J_{\mathrm{H}}<0\right)$.

\subsection{Summary of preliminary results}

Before proceeding further, let us briefly summarize our previous numerical results published in reference [15]. This work was devoted to the comparison of the ground-state properties of the quantum antiferromagnetic spin-1/2 and spin-1 Ising-Heisenberg model on diamond-like decorated planar lattices and its semi-classical Ising analogue. The obtained results revealed that the investigated model system has a rich ground-state phase diagram, which consists of a semi-classically ordered ferrimagnetic phase $\mathrm{FRI}_{1}$ with the perfect antiparallel alignment between the nearestneighbouring Ising and Heisenberg spins and two ferrimagnetic phases $\mathrm{FRI}_{2}$ and $\mathrm{FRI}_{3}$, which differ one from another just in a quantum entanglement of the Heisenberg spin pairs described by the antisymmetric wave function $(|1,0\rangle-|0,1\rangle) / \sqrt{2}$ that emerges just in $\mathrm{FRI}_{2}$. The latter ferrimagnetic phase $\mathrm{FRI}_{3}$ represents the classical ferrimagnetic phase, where the decorating spin pairs may reside in one of the two possible spin states: either $|1,0\rangle$ or $|0,1\rangle$. According to this ambiguous order of the decorating spins, one may regard the phase $\mathrm{FRI}_{3}$ as a partly degenerate state with the non-zero residual entropy $S_{0} / N_{\text {tot }} k_{\mathrm{B}}=[q /(2 q+2)] \ln 2$, which is proportional to the coordination number $q$ of the lattice (total number of decorating spin pairs). Finally, several geometrically frustrated phases can also be found in the ground state depending on whether the hybrid quantum-classical Ising-Heisenberg model or its semi-classical Ising version is considered. In the former case, the ground state is formed by a frustrated phase FRU, where all nodal Ising spins are frustrated due to a quantum superposition of three spin states $|0,0\rangle,|1,-1\rangle$ and $|-1,1\rangle$ of the decorating Heisenberg spins, whose relative probabilities depend on a mutual ratio between interaction parameters. On the other hand, two different frustrated phases $\mathrm{FRU}_{1}$ and $\mathrm{FRU}_{2}$ can be detected for the semi-classical Ising analogue of the model. In the former phase $\mathrm{FRU}_{1}$, the geometric frustration of the nodal Ising spins is caused by "non-magnetic" nature of the Heisenberg spin 
dimers $|0,0\rangle$, while the geometric frustration in the latter phase $\mathrm{FRU}_{2}$ comes from antiferromagnetic spin states (either $|1,-1\rangle$ or $|-1,1\rangle$ ) of the Heisenberg spin pairs. As it has been shown in reference [15], both these phases can be regarded as special limiting cases of the unique frustrated phase FRU.

\subsection{Finite-temperature behaviour of the semi-classical Ising model}

Now, let us proceed to the discussion of the finite-temperature behaviour of the antiferromagnetic spin-1/2 and spin-1 Ising-Heisenberg model on diamond-like decorated planar lattices. To enable a direct comparison with the ground-state analysis published in reference [15], we start first with the discussion of finite-temperature phase diagrams, which are displayed in figure 2 in the reduced units $t=k_{\mathrm{B}} T /\left|J_{\mathrm{I}}\right|, d=D /\left|J_{\mathrm{I}}\right|$ and $\alpha=\left|J_{\mathrm{H}}\right| /\left|J_{\mathrm{I}}\right|$, describing the dimensionless temperature, the relative strength of the AZFS parameter and the strength of the Heisenberg interaction normalized with respect to the Ising interaction, respectively. This figure shows the critical temperature of the semi-classical Ising version of the investigated mixed-spin model on diamond-like decorated honeycomb (figure 2a), square (figure 2b) and triangular (figure 2r) lattices as a function of the AZFS parameter $d$ for several values of the interaction ratio $\alpha$. Note that solid lines depicted in this figure are the unique solutions of the critical conditions (2.3) and, as a consequence, they represent the lines of the second-order phase transitions separating the spontaneously ordered phases $\left(\mathrm{FRI}_{1}\right.$ or $\left.\mathrm{FRI}_{3}\right)$ from the disordered paramagnetic one. As one can clearly see from figure 2, the overall critical behaviour of the system very sensitively depends on the strength of the interaction parameters $\alpha$ and $d$, as well as, the topology (coordination number) of the lattice; for $\alpha<1$, the critical temperature $t_{c}$ either monotonously decreases upon decrease of the AZFS parameter until it tends towards zero temperature at the boundary value $d=-1$ (see the curves labeled as $\alpha=0.1$ and 0.5 ), or it exhibits an interesting non-monotonous dependence to be closely related to the $\mathrm{FRI}_{1} \rightarrow \mathrm{FRI}_{3}$ phase transition when the interaction ratio $\alpha$ is sufficiently close to the value $\alpha=1$ (see e.g. the case $J_{\mathrm{H}} / J_{\mathrm{I}}=0.9$ and figure $2(\mathrm{a})$ in reference [15] for clarity). On the other hand, if one considers $1 \leqslant \alpha \leqslant 2$, the non-zero critical temperature may be observed just around the interval of the AZFS parameters $d \in(-1,1-\alpha)$, where the partly degenerate ferrimagnetic phase $\mathrm{FRI}_{3}$ constitutes the ground state. Furthermore, several interesting regions with reentrant phase transitions can also be observed in finite-temperature phase diagrams shown in figure 2. More specifically, the diamond-like decorated honeycomb lattice exhibits the reentrant behaviour with either two or three consecutive critical points before the critical line tends to zero temperature when the interaction ratio $\alpha$ acquires very small values, i.e., when the antiferromagnetic interaction $J_{\mathrm{H}}$ between decorating spins is much less than the competitive antiferromagnetic interaction $J_{\mathrm{I}}$ between the nodal and decorating spins (see the detail of figure $2 \mathrm{a}$ ). This non-trivial behaviour quickly vanishes as the competitive interaction $J_{\mathrm{H}}$ strengthens (see the cases labeled as $\alpha=0.01$, 0.05 and 0.1 ), which suggests that it appears just as a result of a mutual competition between the interaction $J_{\mathrm{I}}$ favouring the antiferromagnetic arrangement of nodal Ising spins with respect to their nearest spin neighbours and the AZFS parameter $D<0$ that tends to lower the spin states of decorating spins. By contrast, other two diamond-like decorated square and triangular lattices exhibit the reentrant behaviour in the left neighbourhood of the boundary value $d=-1$, where the frustrated phase $\mathrm{FRU}_{1}$ constitutes the ground state, but for any $\alpha \in(0,2)$ (see figures 2 b and c). In these parts of finite-temperature phase diagrams, both the lattices start from the disordered ground state $\mathrm{FRU}_{1}$ before entering the partly ordered phase $\mathrm{FRI}_{3}$ at lower critical temperature $t_{\mathrm{c} 1}$, whose spontaneous order disappears due to strong thermal fluctuations at the upper critical temperature $t_{\mathrm{c} 2}$. Note that the observed non-trivial behaviour fully corresponds to the condition that the coexistence of the spin order and disorder in the ground state of frustrated Ising systems leads to the occurrence of the reentrant phenomenon at non-zero temperatures 34. However, this condition is only necessary but not sufficient for the occurrence of the reentrant phenomenon in Ising systems, which can be directly seen from the regions around the boundary points $d=1-\alpha$ $(\alpha \geqslant 1)$ and $d=\alpha-1(\alpha<1)$ in figures $2 \mathrm{~b}$, c. Obviously, just the square and triangular lattices show the reentrance in these parts of phase diagrams (see details of figures $2 \mathrm{~b}$ and $\mathrm{c}$ ). As the detailed ground-state analysis has revealed, the ground-state degeneracy of the ferrimagnetic phase 

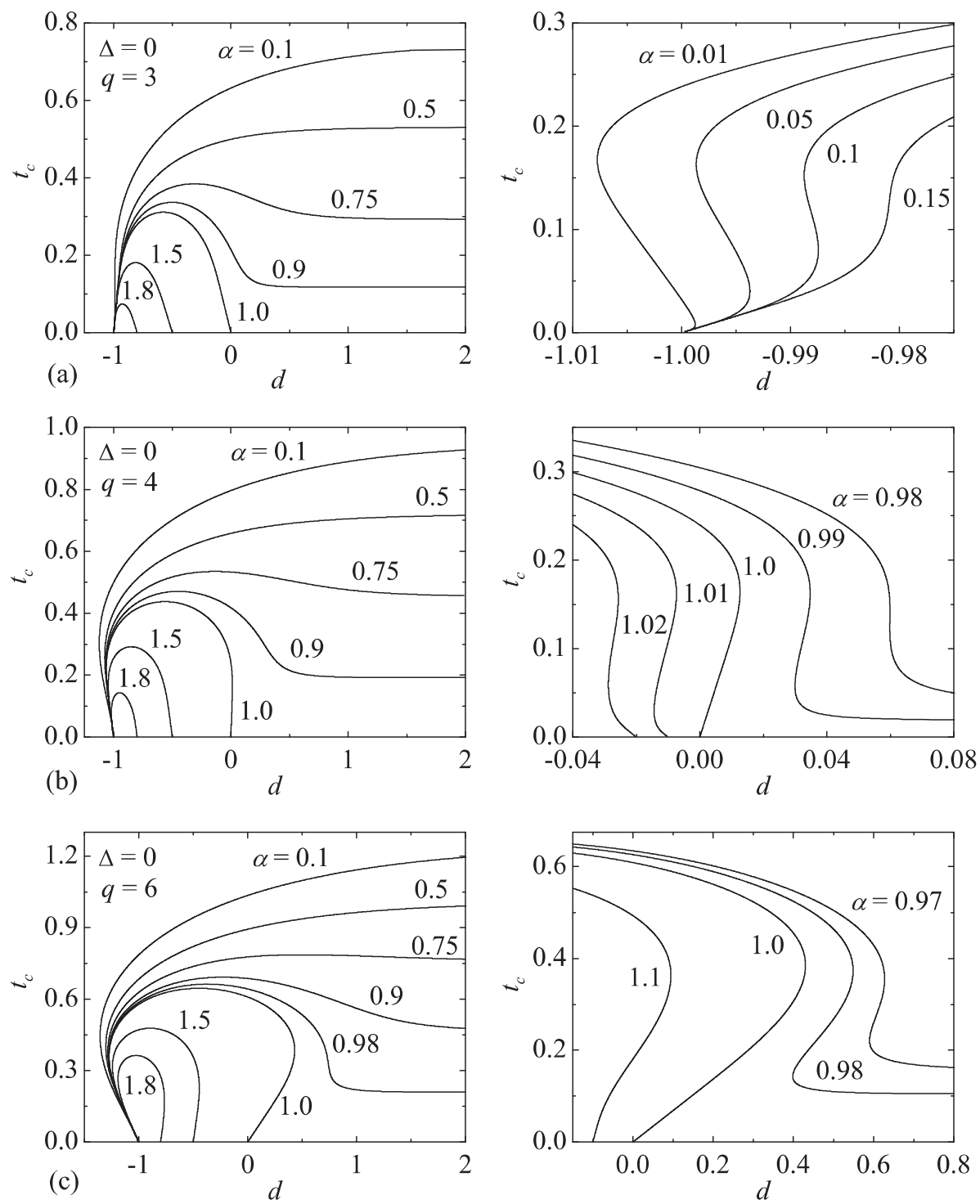

Figure 2. The critical temperature of the semi-classical Ising limit of the mixed spin- $1 / 2$ and spin1 Ising-Heisenberg model on diamond-like decorated honeycomb (figure $2 \mathrm{~b}$ ), square (figure 2 $\mathrm{b}$ ) and triangular (figure 25) lattices as a function of the AZFS parameter $d$ for several values of the interaction ratio $\alpha$. The right column illustrates in detail the regions, where the system exhibits reentrant phase transitions with two or three consecutive critical points. 
$\mathrm{FRI}_{3}$ is not high enough in order to cause the reentrant phenomenon in the case of honeycomb lattice (remember that the residual entropy of the honeycomb lattice is $S_{0} / N_{\text {tot }} k_{\mathrm{B}}=0.2599$, while the residual entropy of the square and triangular lattices is $S_{0} / N_{\text {tot }} k_{\mathrm{B}}=0.2773$ and 0.2971 , respectively). Actually, the square lattice exhibits the reentrant behaviour only if competitive exchange
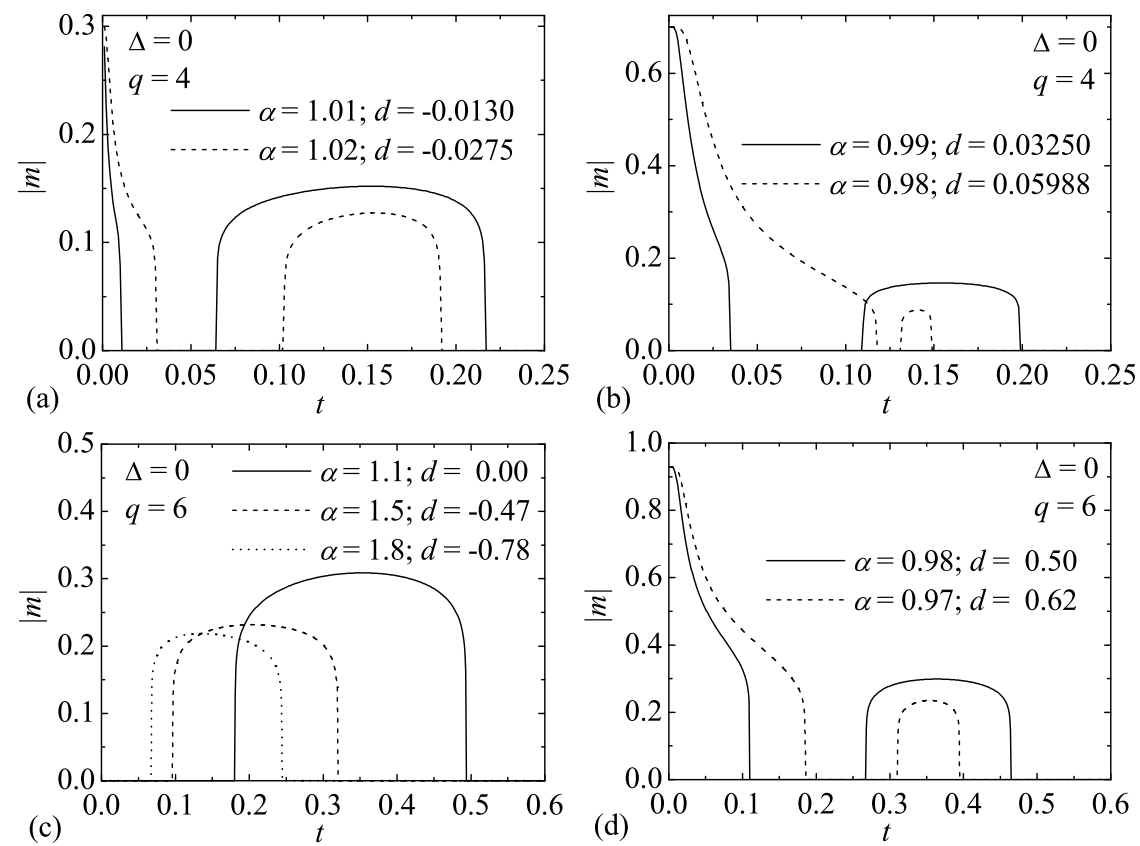

Figure 3. Temperature dependencies of the total magnetization of the decorated square (figures $3 \mathrm{a}, \mathrm{b}$ ) and triangular (figures $3 \mathrm{k}, \mathrm{d}$ ) lattices for such combination of the interaction parameters $\alpha$ and $d$, where the system exhibits the reentrant behaviour.

interactions between the nearest-neighbouring spins are equal or approximately equal to each other (i.e. if $\alpha \simeq 1$ ). On the other hand, the triangular lattice shows the reentrance in this region for the interaction ratio $\alpha \lesssim 1$ as well as for all $\alpha \in\langle 1,2$ ) (see figure 2 c and its detail). It is noteworthy that the region with reentrant phase transitions gradually vanishes as the difference between the considered ratio $\alpha$ and the value 1 increases. These results can also be convincingly evidenced by thermal dependencies of the absolute value of the total magnetization $|m|$ displayed in figure 3 for diamond-like decorated square (figure $3 \mathrm{a}, \mathrm{b}$ ) and triangular (figure $3 \mathrm{k}, \mathrm{d}$ ) lattices.

\subsection{Finite-temperature behaviour of the isotropic quantum version of the model}

To provide a deeper insight into the finite-temperature behaviour of the quantum version of the investigated model system, let us turn our attention to the phase diagrams displayed in figure 4. This figure shows the critical temperature of the mixed spin- $1 / 2$ and spin- 1 Ising-Heisenberg model on diamond-like decorated honeycomb, square and triangular lattices as a function of the interaction ratio $\alpha$ and the AZFS parameter $d$ for the fixed the exchange anisotropy $\Delta=1$. Apart from the expected monotonous decrease of $t_{\mathrm{c}}$ with the increasing ratio $\alpha$ and/or decreasing $d$, one may also find here more interesting non-monotonic dependencies of $t_{\mathrm{c}}$ as well as several striking regions with reentrant phase transitions. More specifically, the honeycomb lattice shows the reentrant behaviour with either two or three consecutive critical points just for very small values of the interaction ratio $\alpha$ and for the particular values of the AZFS paremeter $d \gtrsim-1$. It is quite obvious that the origin of this non-trivial phenomenon lies in a mutual competition between the antiferromagnetic Ising interaction $J_{\mathrm{I}}$ and the easy-plane AZFS parameter $D<0$. As could be expected, the parameter region with reentrant phase transitions enlarges with the increasing coor- 

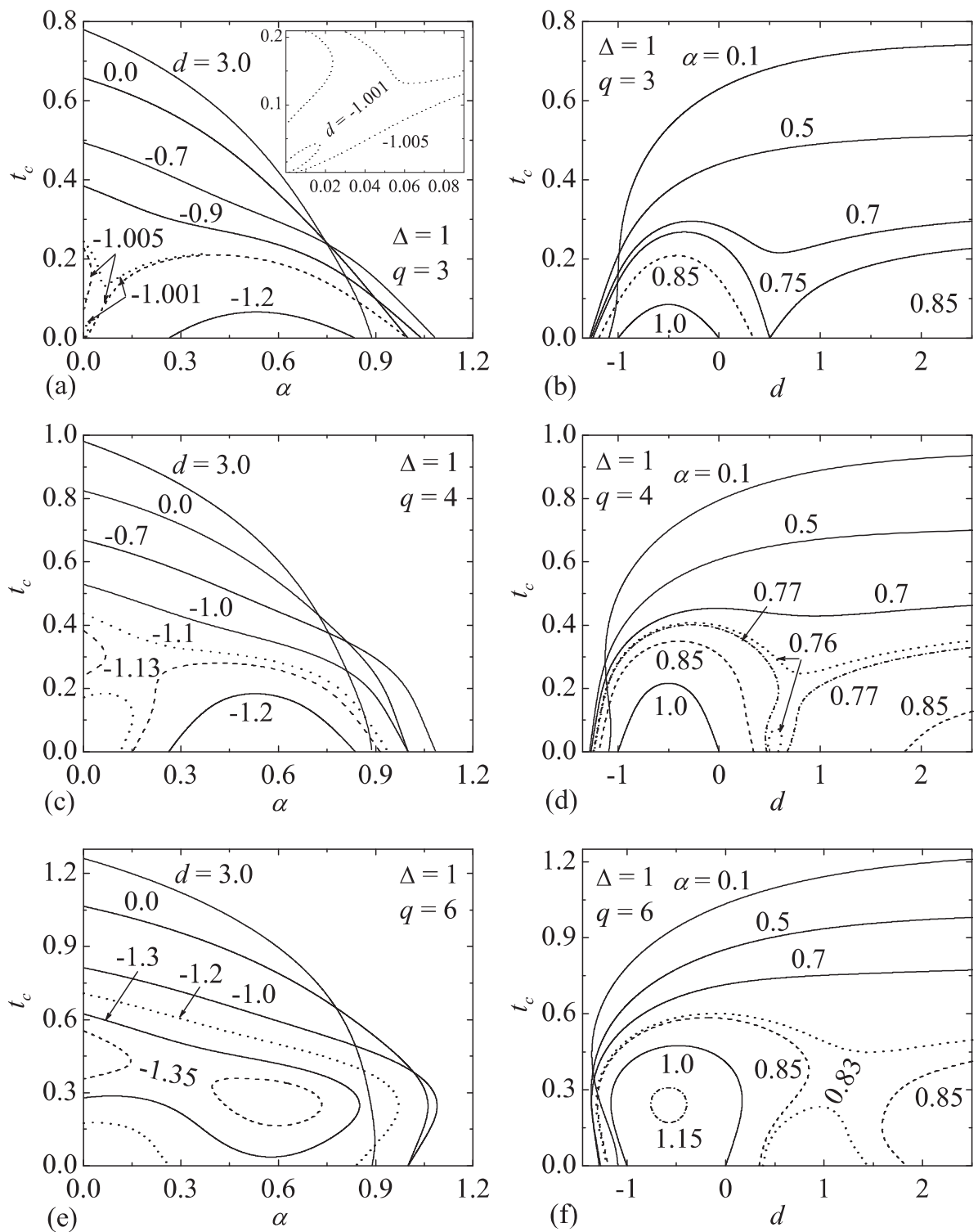

Figure 4. The critical temperature of the mixed spin- $1 / 2$ and spin- 1 Ising-Heisenberg model on diamond-like decorated honeycomb (figures $4 \mathrm{k}, \mathrm{b}$ ), square (figures 4 $4 \mathrm{r}, \mathrm{d}$ ) and triangular (figures 4, f) lattices the fixed the exchange anisotropy $\Delta=1$ as a function of the interaction ratio $\alpha$ for several values of the AZFS parameter $d$ and as a function of the AZFS parameter $d$ for several values of $\alpha$. 
dination number of the lattice. Indeed, this becomes quite clear from figures 4 r and e, the spin-1/2 and spin-1 Ising-Heisenberg model on the decorated square and triangular lattices exhibits two or three critical points for $D>0$ and $J_{\mathrm{H}}(\Delta)$, whereas the effect of the former interaction parameter is supported by the Ising interaction $J_{\mathrm{I}}$. Finally, the remarkable parts of finite-temperature phase diagrams represent particular dependencies of $t_{\mathrm{c}}$ forming closed loops (see figure 2 in reference [35]). In these regions of phase diagrams, the system starts with the disordered ground state before entering the spontaneously ordered ferrimagnetic phase $\mathrm{FRI}_{2}$ at lower critical temperature $t_{\mathrm{c} 1}$, which subsequently disappears due to strong thermal fluctuations at upper critical temperature $t_{\mathrm{c} 2}$ (see the curves $d=-1.35$ in figure 4 e and $\alpha=1.15$ in figure 4 f). The results displayed in figure 4 can
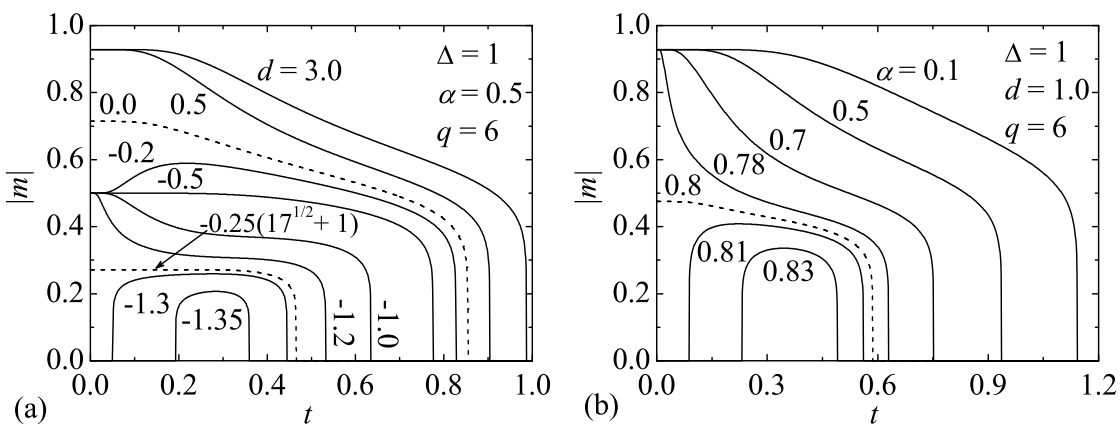

Figure 5. Temperature dependencies of the total magnetization of the mixed-spin IsingHeisenberg model on diamond-like decorated triangular lattice with the fixed the exchange anisotropy $\Delta=1$ for the interaction ratio $\alpha=0.5$ and several values of the AZFS parameter $d$ (figure $5 \mathrm{k}$ ) and for the AZFS parameter $d=1$ and several values of the ratio $\alpha$. The dependencies depicted as broken curves correspond to the two-phase coexistence at $t=0$ (see the discussion).

be also convincingly evidenced by thermal dependencies of the total magnetization. Since the most diverse finite-temperature behaviour has been observed for the diamond-like decorated triangular lattice, in figure 5 we have depicted some typical temperature variations of the absolute value of total magnetization $|m|$ for this particular model system. As one can clearly see, the magnetization exhibits reentrant transitions with two consecutive critical points for interaction ratio $\alpha=0.5$ and the AZFS parameters $d \lesssim-0.25(\sqrt{17}+1)$ as depicted in figure 5 a or for the particular case of the AZFS parameter $d=1$ and the interaction ratio $\alpha \gtrsim 0.8$ as depicted in figure $5 \mathrm{~b}$. Note that these values of the interaction parameters $\alpha$ and $d$ are from a close vicinity of the ground-state phase transition between the ferrimagnetically ordered phase $\left(\mathrm{FRI}_{1}\right.$ or $\left.\mathrm{FRI}_{2}\right)$ and the disordered $\mathrm{FRU}$ phase. On the other hand, if one considers the parameter space $d>-0.25(\sqrt{17}+1)$ for the interaction ratio $\alpha=0.5$ or $\alpha<0.8$ for the AZFS parameter $d=1$, then the displayed magnetization curves $|m(t)|$ exhibit a single critical point only (see, e.g., the curves $d=-0.5,0.5$ in figure 5 and $\alpha=0.1,0.5,0.7$ in figure $5 \mathrm{~b}$ ).

\section{Concluding remarks}

In the present paper, the critical behaviour of the mixed spin- $1 / 2$ and spin- 1 Ising-Heisenberg model on diamond-like decorated honeycomb, square and triangular lattices has been investigated within the framework of the generalized decoration-iteration mapping transformation. Using this rigorous procedure, the exact solution for the investigated mixed-spin model has been obtained by establishing a precise mapping equivalence with the spin- $1 / 2$ Ising model on the corresponding undecorated planar lattice with the known exact solution. 
The main emphasis of this work was laid upon the systematic study of the finite-temperature phase diagrams that basically depend on the lattice topology and upon the comparison of the critical behaviour of the hybrid quantum-classical Ising-Heisenberg model and its semi-classical Ising variant. The most interesting result consists in the exact evidence of the existence of reentrant phase transitions with either two or three consecutive critical points. The presence of this non-trivial phenomenon has also been evidenced by temperature dependencies of the total magnetization. It has been proved that the higher the coordination number of the lattice is, the more pronounced reentrance is and the more diverse critical behaviour may be found.

Finally, it is worthwhile to remark that even though our theoretical investigation of the mixed spin-1/2 and spin-1 Ising-Heisenberg model on diamond-like decorated planar lattices has been mainly aimed at providing a deeper insight into cooperative and quantum features of this exactly solvable model, we hope that our results might stimulate research on possible experimental realizations of this interesting quantum spin model and confirm our theoretical predictions. From this perspective, the most promising approach in experimental realizations of our model system might represent a targeted design of cyano-based polymeric coordination compounds or their isostructural analogues. For instance, the series of bimetallic polymeric coordination compounds $\{\mathrm{Cu}(\mathrm{L})\}_{3}\left[\mathrm{Fe}(\mathrm{CN})_{6}\right]\left(\mathrm{ClO}_{4}\right)_{2} \cdot \mathrm{nH}_{2} \mathrm{O}, \mathrm{L}=\mathrm{N}$-(3-aminopropyl $)-1,3$-propanediamine [35, 36] or N-(2-aminoethyl)-1,3 -propanediamine [37], which possess the diamond-like decorated honeycomb network structure, may represent a useful starting point for this rational synthesis. In this series, the divalent $\mathrm{Cu}^{\mathrm{II}}$ and $\mathrm{Fe}^{\mathrm{II}}$ metal atoms reside decorating and nodal sites of the diamond-like decorated honeycomb lattice, respectively (see figure 1 $1 \mathrm{a}$ ). Unfortunately, the divalent $\mathrm{Fe}^{\mathrm{II}}$ atoms are due to a strong ligand field of the cyano group in the diamagnetic low-spin state with $S=0$. Similarly, the bimetallic polymeric coordination compounds $\left[\mathrm{W}\left\{(\mathrm{CN})_{4} \mathrm{Fe}\left(\mathrm{H}_{2} \mathrm{O}\right)_{2}\right\}_{2}\right] \cdot \mathrm{nH}_{2} \mathrm{O}[38]$ and $\left[\mathrm{W}\left\{(\mathrm{CN})_{4} \mathrm{Co}\left(\mathrm{H}_{2} \mathrm{O}\right)_{2}\right\}_{2}\right] \cdot \mathrm{nH}_{2} \mathrm{O}$ [39] with the diamond-like decorated square network structure (see figure 10) have also been reported quite recently, but the tetravalent $\mathrm{W}^{\mathrm{IV}}$ metal atoms residing nodal sites of the diamond-like decorated square lattice are diamagnetic due to a strong ligand field of the cyano group in those systems.

\section{Acknowledgements}

This work was partially supported by the internal grant of P.J. Safárik University under the contract No. VVGS 1/10-11 and by European Union European regional development fond (ERDF EU) grant under the contract No. ITMS26220120005 (activity 3.2.).

\section{References}

1. Lhuillier C., Misguich G., in: High Magnetic Fields: Applications in Condensed Matter Physics and Spectroscopy, Edited by Berthier C., Lévy L.P., Martinez G., Springer-Verlag, Berlin, 2002; doi:10.1007/3-540-45649-X_6

2. Richter J., Schulenburg J., Honecker A., Quantum Magnetism, Lecture Notes in Physics, Vol. 645, Edited by Schollwöck U., Richter J., Farnell D.J.J., Bishop R.F., Springer-Verlag, Berlin, 2004; doi:10.1007/BFb0119592

3. Misguich G., Lhuillier C., Frustrated Spin Systems, Edited by Diep H.T., World Scientific, Singapore, 2004.

4. Villian J., J. Phys. C: Solid State Phys., 1977, 10, 1717; doi:10.1088/0022-3719/10/10/014

5. Villain J. et al., J. Physique, 1980, 41, 1263; doi:10.1051/jphys:0198000410110126300

6. Zhitomirsky M.E., Phys. Rev. B, 2003, 67, 104421; doi:10.1103/PhysRevB.67.104421.

7. Honecker A., Richter J., Condens. Matter Phys., 2005, 8, 813.

8. Derzhko O., Richter J., Eur. Phys. J. B, 2006, 52, 23; doi:10.1140/epjb/e2006-00273-y.

9. Schnack J., Schmidt R., Richter J., Phys. Rev. B, 2007, 76, 054413; doi:10.1103/PhysRevB.76.054413

10. Honecker A., Schulenburg J., Richter J., J. Phys.: Condens. Matter, 2004, 16, S749; doi:10.1088/0953-8984/16/11/025

11. Strečka J., Jaščur M., Phys. Status Solidi B, 2002, 233, R12; doi:10.1002/1521-3951(200210)233:3<R12::AID-PSSB999912>3.0.CO;2-2

12. Canová L., Strečka J., Jaščur M., Czech. J. Phys., 2004, 54, D579. 
13. Strečka J., Jaščur M., Acta Phys. Slovaca, 2006, 56, 65.

14. Čanová L., Strečka J., Jaščur M., J. Phys.: Condens. Matter, 2006, 18, 4967; doi:10.1088/0953-8984/18/20/020.

15. Canová L., Strečka J., Dely J., Jaščur M., Acta Phys. Pol., 2008, 113, 449.

16. Jaščur M., Strečka J., Čanová L., Acta Phys. Pol., 2008, 113, 453.

17. Strečka J., Čanová L., Lučivjanský T., Jaščur M., J. Phys.: Conf. Ser., 2009, 145, 012058; doi:10.1088/1742-6596/145/1/012058.

18. Canová L., Strečka J., Lučivjanský T., Condens. Matter Phys., 2009, 12, 353.

19. Čanová L., Strečka J., Phys. Status Solidi B, 2010, 247, 433; doi:10.1002/pssb.200945444.

20. Fisher M.E., Phys. Rev., 1959, 113, 969; doi:10.1103/PhysRev.113.969

21. Syozi I., Phase Transition and Critical Phenomena, Vol. 1, Edited by Domb C., Green M.S., Academic Press, New-York, 1972.

22. Rojas O., Valverde J.S., de Sousa S.M., Physica A, 2009, 388, 1419; doi:10.1016/j.physa.2008.12.063.

23. Rudowicz C., Physica B, 2008, 403, 1882; doi:10.1016/j.physb.2007.10.219

24. Rudowicz C., Physica B, 2008, 403, 2312; doi:10.1016/j.physb.2007.12.011

25. Houtappel R.M.F., Physica (Amsterdam), 1950, 16, 425; doi:10.1016/0031-8914(50)90130-3

26. Onsager L., Phys. Rev., 1944, 65, 117; doi:10.1103/PhysRev.65.117

27. Baxter R.J., Choy T.C., Proc. R. Soc. London, Ser. A, 1989, 423, 279; doi:10.1098/rspa.1989.0055

28. Barry J.H., Khatun M., Tanaka T., Phys. Rev. B, 1988, 37, 5193; doi:10.1103/PhysRevB.37.5193.

29. Khatun M., Barry J.H., Tanaka T., Phys. Rev. B, 1990, 42, 4398; doi:10.1103/PhysRevB.42.4398

30. Barry J.H., Tanaka T., Khatun M., Múnera C.H., Phys. Rev. B, 1981, 44, 2595; doi:10.1103/PhysRevB.44.2595.

31. Callen H.B., Phys. Lett., 1963, 4, 161; doi:10.1016/0031-9163(63)90344-5

32. Suzuki M., Phys. Lett., 1965, 19, 267; doi:10.1016/0031-9163(65)90978-9

33. Balcerzak T., J. Magn. Magn. Mater., 2002, 246, 213; doi:10.1016/S0304-8853(02)00056-2

34. Diep H.T., Giacomini H., Frustrated Spin Systems, Edited by Diep H.T., World Scientific, Singapure, 2004.

35. Hong Ch.S., You Y.S., Inorg. Chim. Acta, 2004, 357, 3271; doi:10.1016/j.ica.2004.04.004

36. Zhang H.-X., et al., J. Organomet. Chem., 2000, 598, 63; doi:10.1016/S0022-328X(99)00679-8

37. Trávniček Z., Smékal Z., Escuer A., Marek J., New J. Chem., 2001, 25, 655; doi:10.1039/b006741p.

38. Pilkington M. et al., J. Solid St. Chem., 2001, 159, 262; doi:10.1006/jssc.2001.9155

39. Herrera J.M. et al., Inorg. Chem., 2003, 42, 7052; doi:10.1021/ic034188+

\title{
Фазові переходи у геометрично фрустрованій змішаній спін-1/2 і спін-1 моделі Ізінга-Гайзенберга на ромбічноподібних декорованих плоских ґратках
}

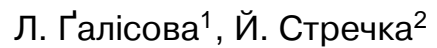 \\ ${ }^{1}$ Механіко-інженерний факультет, Технічний університет, Кошіце, Словацька республіка \\ 2 Природничий факультет, Університет ім. П.Й. Шафарика, Кошіце, Словацька республіка
}

Досліджуються фазові переходи у змішаній спін-1/2 і спін-1 моделі Ізінга-Гайзенберга на декількох декорованих плоских ґратках, що складаються зі сполучених ромбів в рамках узагальненого декораційно-ітераційного перетворення. Основна увага приділяється систематичному вивченню фазових діаграм при скінчених температурах в залежності від топології гратки. Критична поведінка гібридної квантово-класичної моделі Ізінга-Гайзенберга порівнюється 3 поведінкою iї напівкласичного ізінгівського аналога. Показано, що обидві моделі на ромбічноподібних декорованих плоских ґратках демонструють цікаву критичну поведінку, включаючи зворотні фазові переходи. Чим вище координаційне число ґратки, тим помітнішою може бути зворотність.

Ключові слова: модель Ізігнга-Гайзенберга, декораційно-ітераційне перетворення, геометрична фрустрація, зворотні фазові переходи 
